\title{
ON STRONG RIESZ SUMMABILITY FACTORS OF INFINITE SERIES. I
}

\author{
J. S. RATTI
}

(1.1) Let $\sum_{n=1}^{\infty} a_{n}$ be a given infinite series, and $\left\{\lambda_{n}\right\}$ an increasing sequence of positive numbers, tending to infinity with $n$. We write

$$
\begin{aligned}
A^{0}(\omega) & =A(\omega)=\sum_{\lambda_{n}<\omega} a_{n}, \\
A^{k}(\omega) & =\sum_{\lambda_{n}<\omega}\left(\omega-\lambda_{n}\right)^{k} a_{n} \\
& =\int_{0}^{\omega}(\omega-t)^{k} d A(t), \\
A^{k}(\omega) & =0 \text { for } \omega \leqq 1, \text { and } k>-1 .
\end{aligned}
$$

The series $\sum a_{n}$ is said to be summable $(R, \lambda, k)$ to the sum $s$, if $\lim \omega^{-k} A^{k}(\omega) \rightarrow s$ as $\omega \rightarrow \infty$. The given series is said to be strongly summable $(R, \lambda, k)$, or simply summable $[R, \lambda, k]$ to the sum $s$, if

$$
\int_{0}^{\omega}\left|x^{-k+1} A^{k-1}(x)-s\right| d x=o(\omega) ;
$$

where $k>0$. The series $\sum a_{n}$ is said to be strongly summable $(R, \lambda, k)$ with index $m>0$, or summable $[R, \lambda, k, m]$ to the sum $s$, if

$$
\int_{0}^{\omega}\left|x^{-k+1} A^{k-1}(x)-s\right|^{m} d x=o(\omega),
$$

where $k>0$ and $k m^{\prime}>1,\left(1 / m+1 / m^{\prime}=1\right)$.

(1.2) The classical second theorem of consistency due to Hardy and Riesz [3] is to the effect that if $\sum a_{n}$ is summable $(R, \lambda, k)$ and $\lambda_{n}=e^{\mu_{n}}$, then it is also summable $(R, \mu, k)$ to the same sum. Later Hardy [2] generalized this theorem and proved:

Theorem A. If the series $\sum a_{n}$ is summable $(R, \lambda, k)$ and $\mu$ is a logarithmico-exponential function (briefly an L-function) of $\lambda$, tending to infinity with $\lambda$, such that $\mu=O\left(\lambda^{\Delta}\right)$, where $\Delta$ is some constant, then $\sum a_{n}$ is summable $(R, \mu, k)$.

Finally Hirst [4] removed the limitation on $\mu$ of being an $L$-function, replacing $\mu$ by a more general function $\phi(t)$, and proved:

Received by the editors September 28, 1966. 
THeOrem B. If $\sum a_{n}$ is summable $(R, \lambda, k)$, then it is summable $(R, \mu, k)$ to the same sum, where $\mu=\phi(\lambda)$, and $\phi(t)$ is a function which increases steadily to infinity with $t$ and is a $(k+1)$ th indefinite integral for $t>0$, such that

$$
\int_{0}^{x} t^{n}\left|\phi^{(n+1)}(t)\right| d t=O\{\phi(x)\} ; \quad n=1,2, \cdots, k .
$$

The following theorem on strong Riesz summability factors was proved by Borwein and Shawyer [1].

Theorem C. For all $k \geqq 1$, if

(i) $\phi(t)$ is an L-function,

(ii) $1 / \omega=O\left\{\phi^{\prime}(\omega) / \phi(\omega)\right\}$,

(iii) $\psi(\omega)=\left\{\phi(\omega) / \omega \phi^{\prime}(\omega)\right\}^{k}$,

then $\sum a_{n} \psi\left(\lambda_{n}\right)$ is summable $[R, \phi(\lambda), k]$ whenever $\sum a_{n}$ is summable $[R, \lambda, k]$.

The object of this paper is to establish a more general summability factor theorem for strong Riesz summability, which includes as a particular case Theorem C.

I should like to express my gratitude to Professor D. Waterman and the referee for their helpful suggestions.

(2.1) In the following we take functions $\phi(t)$ and $\psi(t)$ to be defined in $(0, \infty)$ and to be as many times differentiable as required. In addition let $\phi(t)$ be nonnegative, monotone increasing, and tending to infinity with $t$.

We establish the following theorem.

THEOREM. Let $k$ be a positive integer, and $\phi(t)$ and $\psi(t)$ be $(k+1)$ th indefinite integrals for $t>0$. If there is a positive, nondecreasing function $\gamma(t)$ such that

(i) $\gamma(t)=O(t)$ in $(a, \infty) ; a>0$,

(ii) $t^{k} \psi^{(n)}(t)=O\left(\{\gamma(t)\}^{k-n}\right) ; n=0,1, \cdots, k ; t>a$,

(iii) $\{\gamma(t)\}^{n} \phi^{(n)}(t)=O[\phi(t)]: n=1,2, \cdots, k ; t>a$, then $\sum a_{n} \psi\left(\lambda_{n}\right)$ is summable $[R, \phi(\lambda), k, m]$, whenever $\sum a_{n}$ is summable $[R, \lambda, k, m]$, where $m \geqq 1$ and $k m^{\prime}>1$.

(2.2) The following lemmas will be required in the proof of our theorem.

Lemma 1 [3]. If $k$ is a positive integer, then $A(t)=(1 / k !)(d / d t)^{k} A^{k}(t)$.

Lemma 2 [5]. If $\sum a_{n}$ is summable $[R, \lambda, k, m]$ for $m \geqq 1$, then it is summable $(R, \lambda, k)$. 
Lemma 3 [8]. If $n$ is a positive integer and $m \neq 0$, then the nth derivative of $\{f(x)\}^{m}$ is a sum of the constant multiples of a finite number of terms of the form

$$
\{f(x)\}^{m-q} \prod_{p=1}^{n}\left\{f^{(p)}(x)\right\}^{\alpha_{p}},
$$

where $1 \leqq q \leqq n$ and the $\alpha$ 's are nonnegative integers such that

$$
\sum_{1}^{n} \alpha_{p}=q \text { and } \sum_{1}^{n} p \alpha_{p}=n
$$

If $m$ is a positive integer, then $1 \leqq q \leqq \min (m, n)$.

3. Proof of the theorem. We may suppose without loss of generality that the sum of the given series is zero. Then the hypothesis of summability $[R, \lambda, k, m]$ of $\sum a_{n}$ reduces to

$$
\int_{0}^{\omega}\left|A^{k-1}(t)\right|^{m} d t=o\left[\omega^{(k-1) m+1}\right] \text {. }
$$

And for summability $[R, \phi(\lambda), k, m]$ of $\sum a_{n} \psi\left(\lambda_{n}\right)$ we must show

$$
\int_{0}^{\omega} \phi^{\prime}(t)\left|B^{k-1}\{\phi(t)\}\right|^{m} d t=o\left[\{\phi(\omega)\}^{(k-1) m+1}\right]
$$

where

$$
B^{k-1}\{\phi(t)\}=\int_{0}^{t}\{\phi(t)-\psi(u)\}^{k-1} \psi(u) d A(u) .
$$

Integrating by parts, we have

$$
B^{k-1}\{\phi(t)\}=-\int_{0}^{t}(\partial / \partial u)\left[\{\phi(t)-\phi(u)\}^{k-1} \psi(u)\right] A(u) d u .
$$

And by Lemma 1 ,

$$
\begin{aligned}
& B^{k-1}\{\phi(t)\} \\
& =-\frac{1}{(k-1) !} \int_{0}^{t} \frac{\partial}{\partial u}\left[\{\phi(t)-\phi(u)\}^{k-1} \psi(u)\right]\left(\frac{d}{d u}\right)^{k-1} A^{k-1}(u) d u .
\end{aligned}
$$

Since $A^{k}(u)$ and its first $(k-1)$ derivatives vanish at $u=0$, integrating (3.4) by parts $(k-1)$ times, we get

$$
\begin{aligned}
B^{k-1}\{\phi(t)\} & =A^{k-1}(t) \psi(t)\left\{\phi^{\prime}(t)\right\}^{k-1} \\
+ & \frac{(-1)^{k}}{(k-1) !} \int_{0}^{t} A^{k-1}(u)\left(\frac{\partial}{\partial u}\right)^{k}\left[\{\phi(t)-\phi(u)\}^{k-1} \psi(u)\right] d u .
\end{aligned}
$$


Thus to prove the theorem, it is sufficient to prove that

$$
\int_{0}^{\omega}\left|A^{k-1}(t)\right|^{m}\{\psi(t)\}^{m}\left\{\phi^{\prime}(t)\right\}^{(k-1) m+1} d t=o\left[\{\phi(\omega)\}^{(k-1) m+1}\right]
$$

and

$$
\begin{gathered}
\int_{0}^{\omega} \phi^{\prime}(t)\left|\int_{0}^{t} A^{k-1}(u)\left(\frac{\partial}{\partial u}\right)^{k}\left[\{\phi(t)-\phi(u)\}^{k-1} \psi(u)\right] d u\right|^{m} d t \\
=o\left[\{\phi(\omega)\}^{(k-1) m+1}\right] .
\end{gathered}
$$

Proof of (3.6). Let

$$
\begin{aligned}
J & =\int_{0}^{\omega}\left|A^{k-1}(t)\right|^{m}\{\psi(t)\}^{m}\left\{\phi^{\prime}(t)\right\}^{(k-1) m+1} d t \\
& =\int_{0}^{1}+\int_{1}^{\omega} .
\end{aligned}
$$

By hypotheses (ii) and (iii) of the theorem and the fact that $A^{k}(\omega)=0$ for $\omega \leqq 1$ and $k>-1$, we have

$$
\begin{aligned}
J & =O\left(\int_{1}^{\omega}\left|A^{k-1}(t)\right|^{m}\left(\frac{\gamma(t)}{t}\right)^{k m}\left(\frac{\phi(t)}{\gamma(t)}\right)^{(k-1) m+1} d t\right) \\
& =O\left(\{\phi(\omega)\}^{(k-1) m+1} \int_{1}^{\omega}\left|A^{k-1}(t)\right|^{m} t^{-k m}\{\gamma(t)\}^{m-1} d t\right) \\
& =O\left(\{\phi(\omega)\}^{(k-1) m+1}\{\gamma(\omega)\}^{m-1} \int_{1}^{\omega}\left|A^{k-1}(t)\right|^{m} t^{-k m} d t\right),
\end{aligned}
$$

since $k \geqq 1, m \geqq 1$ and $\gamma(t)$ is a nondecreasing positive function.

Integrating the last expression by parts and using (3.1) we have:

$$
\begin{aligned}
J & =o\left[\{\phi(\omega)\}^{(k-1) m+1}\{\gamma(\omega)\}^{m-1} \omega^{1-m}\right] \\
& =o\left[\{\phi(\omega)\}^{(k-1) m+1}\right]
\end{aligned}
$$

by (i) of the theorem.

Proof of (3.7). Note that $(\partial / \partial u)^{k}\left[\{\phi(t)-\phi(u)\}^{k-1} \psi(u)\right]$ is a sum of constant multiples of terms of type

$$
\psi^{(\nu)}(u)(\partial / \partial u)^{k-\nu}\{\phi(t)-\phi(u)\}^{k-1} ; \quad 0 \leqq \nu \leqq k,
$$

and, by Lemma $3,(\partial / \partial u)^{k-\nu}\{\phi(t)-\phi(u)\}^{k-1}$ is a linear combination of expressions of the form

$$
\{\phi(t)-\phi(u)\}^{k-1-\mu} \prod_{p=1}^{k-\nu}\left\{\phi^{(p)}(u)\right\}^{\alpha_{p}}
$$


where $0 \leqq \mu \leqq k-\nu-1$ and

$$
\sum_{p=1}^{k-\nu} \alpha_{p}=\mu, \quad \sum_{p=1}^{k-\nu} p \alpha_{p}=k-\nu .
$$

Thus to prove (3.7) it is enough to show that

$$
\begin{gathered}
\int_{0}^{\omega} \phi^{\prime}(t)\left|\int_{0}^{t} A^{k-1}(u)\{\phi(t)-\phi(u)\}^{k-1-\mu} \psi^{(\nu)}(u) \prod_{p=1}^{k-\nu}\left\{\phi^{(p)}(u)\right\}^{\alpha_{p}} d u\right|^{m} d t \\
=o\left[\{\phi(\omega)\}^{(k-1) m+1}\right] .
\end{gathered}
$$

Consider

$$
f(t)=\int_{0}^{t} A^{k-1}(u)\{\phi(t)-\phi(u)\}^{k-1-\mu} \psi^{(\nu)}(u) \prod_{p=1}^{k-\nu}\left\{\phi^{(p)}(u)\right\}^{\alpha_{p}} d u .
$$

Integrating (3.9) by parts and making use of the fact that summability $[R, \lambda, k, m]$ implies summability $(R, \lambda, k)$ we have

$$
\begin{aligned}
f(t)= & {\left[o\left(u^{k}\right) \psi^{(\nu)}(u)\{\phi(t)-\phi(u)\}^{k-1-\mu} \prod_{p=1}^{k-\nu}\left\{\phi^{(p)}(u)\right\}^{\alpha_{p}}\right]_{0}^{t} } \\
& +o\left(\int_{0}^{t} u^{k} \frac{d}{d u}\left[\psi^{(\nu)}(u)\{\phi(t)-\phi(u)\}^{k-1-\mu} \prod_{p=1}^{k-\nu}\left\{\phi^{(p)}(u)\right\}^{\alpha_{p}}\right] d u\right) \\
= & I_{1}+I_{2} \text {, say. }
\end{aligned}
$$

When $0 \leqq \mu<k-1$, then $I_{1}=0$; and when $\mu=k-1$,

$$
I_{1}=\left[o\left(t^{k}\right) \psi^{(\nu)}(t) \prod_{p=1}^{k-\nu}\left\{\phi^{(p)}(t)\right\}^{\alpha_{p}}\right]
$$

Using hypotheses (ii) and (iii) and the relations

$$
\sum_{1}^{k-\nu} p \alpha_{p}=k-\nu, \quad \sum_{1}^{k-\nu} \alpha_{p}=\mu
$$

we get

$$
\begin{aligned}
I_{1} & =o\left(\prod_{p=1}^{k-\nu}\left[\{\gamma(t)\}^{p}\left\{\phi^{(p)}(t)\right\}\right]^{\alpha_{p}}\right) \\
& =o\left(\{\phi(t)\} \Sigma \alpha_{p}\right) \\
& =o\left(\{\phi(t)\}^{\mu}\right) .
\end{aligned}
$$

Thus 


$$
I_{1}=o\left(\{\phi(t)\}^{k-1}\right) .
$$

Now $I_{2}=I_{3}+I_{4}+I_{5}$, where

$$
\begin{aligned}
& I_{3}=o\left(\int_{0}^{t} u^{k}\{\phi(t)-\phi(u)\}^{k-2-\mu} \phi^{\prime}(u) \psi^{(v)}(u) \prod_{p=1}^{k-\nu}\left\{\phi^{(p)}(u)\right\}^{\alpha_{p}} d u\right), \\
& I_{4}=o\left(\int_{0}^{t} u^{k}\{\phi(t)-\phi(u)\}^{k-1-\mu} \psi^{(\nu+1)}(u) \prod_{p=1}^{k-\nu}\left\{\phi^{(p)}(u)\right\}^{\alpha_{p}} d u\right), \\
& I_{5}=o\left(\int_{0}^{t} u^{k}\{\phi(t)-\phi(u)\}^{k-1-\mu} \psi^{(\nu)}(u) \sum_{p=1}^{k-\nu} \alpha_{p} \frac{\phi^{(p+1)}(u)}{\phi^{p}(u)} \prod_{r=1}^{k-\nu}\left\{\phi^{(r)}(u)\right\}^{\alpha_{r}} d u\right) .
\end{aligned}
$$

By hypothesis (ii),

$$
\begin{aligned}
I_{3} & =o\left(\int_{0}^{t}\{\phi(t)-\phi(u)\}^{k-\mu-2} \phi^{\prime}(u) \prod_{p=1}^{k-\nu}\left[\{\gamma(u)\}^{p} \phi^{(p)}(u)\right]^{\alpha_{p}} d u\right) \\
& =o\left(\{\phi(t)\}^{\mu} \int_{0}^{t}\{\phi(t)-\phi(u)\}^{k-\mu-2} \phi^{\prime}(u) d u\right) .
\end{aligned}
$$

Thus

$$
I_{3}=o\left(\{\phi(t)\}^{k-1}\right) \text {. }
$$

Consider $I_{4}$ (a) If all $\alpha_{p}$ 's are zero then $\mu=0$ and $k=\nu$, since $\sum \alpha_{p}=\mu$ and $\sum p \alpha_{p}=k-\nu$. Thus

$$
\begin{aligned}
I_{4} & =o\left(\int_{0}^{t} u^{k}\{\phi(t)-\phi(u)\}^{k-1} \psi^{(k+1)}(u) d u\right) \\
& =o\left(t^{k}\{\phi(t)\}^{k-1} \int_{0}^{t} \psi^{(k+1)}(u) d u\right) \\
& =o\left(\{\phi(t)\}^{k-1}\right),
\end{aligned}
$$

since $t^{k} \psi^{(k)}(t)=O(1)$ by (ii).

(b) Suppose that not all $\alpha_{p}$ 's are zero, so that $\mu \geqq 1$. If $\alpha_{q} \geqq 1$, we have, by (ii) and (iii)

$$
\begin{aligned}
I_{4} & =o\left(\{ \phi ( t ) \} ^ { \mu - 1 } \int _ { 0 } ^ { t } \left\{\phi(t)-\phi(u)\left\{{ }^{k-1-\mu}\{\gamma(u)\}^{q-1} \phi^{(q)}(u) d u\right)\right.\right. \\
& =o\left(\{\phi(t)\}^{k-2}\{\gamma(t)\}^{q-1} \int_{0}^{t} \phi^{(q)}(u) d u\right) \\
& =o\left(\{\phi(t)\}^{k-2}\left[\{(t)\}^{q-1} \phi^{(q-1)}(t)\right]\right) \\
& =o\left(\{\phi(t)\}^{k-1}\right) .
\end{aligned}
$$

Thus 


$$
I_{4}=o\left(\{\phi(t)\}^{k-1}\right) .
$$

When $k>1$,

$$
\begin{aligned}
I_{5}=o\left(\int_{0}^{t}\{\phi(t)-\phi(u)\}^{k-1-\mu} \sum_{p=1}^{k-\nu} \alpha_{p}\{\gamma(u)\}^{p}\left|\phi^{(p+1)}(u)\right|\right. \\
\left.\cdot \prod_{r=1}^{k-\nu}\left|\{\gamma(u)\}^{r} \phi^{(r)}(u)\right|^{\alpha_{r}} d u\right),
\end{aligned}
$$

where $\prod^{(p)}$ means that in the $p$ th factor the power is $\alpha_{p}-1$ (not $\left.\alpha_{p}\right)$. Hence

$$
\begin{aligned}
I_{5} & =o\left(\int_{0}^{t}\{\phi(t)-\phi(u)\}^{k-1-\mu} \sum_{p=1}^{k-\nu}\{\gamma(u)\}^{p} \phi^{(p+1)}(u)\{\phi(u)\}^{\Sigma_{\alpha_{p}-1} d u}\right) \\
& =o\left(\{\phi(t)\}^{k-2} \sum_{p=1}^{k-\nu}\{\gamma(t)\}^{p} \int_{0}^{t} \phi^{(p+1)}(u) d u\right) \\
& =o\left(\{\phi(t)\}^{k-2} \sum_{p=1}^{k-\nu}\left[\{\gamma(t)\}^{p} \phi^{(p)}(t)\right]\right) \\
& =o\left(\{\phi(t)\}^{k-1}\right) .
\end{aligned}
$$

When $k=1$, the proof is similar to that for $I_{4}$, case (a). Thus

$$
I_{5}=o\left(\{\phi(t)\}^{k-1}\right) .
$$

From (3.10), (3.11), (3.12) and (3.13) it follows that the function $f(t)$ of $(3.9)$ is $o\left(\{\phi(t)\}^{k-1}\right)$. Thus

$$
\begin{aligned}
\int_{0}^{\omega} \phi^{\prime}(t)|f(t)|^{m} d t & =o\left(\int_{0}^{\omega}\{\phi(t)\}^{(k-1) m} \phi^{\prime}(t) d t\right) \\
& =o\left[\{\phi(\omega)\}^{(k-1) m+1}\right] .
\end{aligned}
$$

This completes the proof of the theorem.

In the special case when $\psi(t)=1, \gamma(t)=t, m=1$, we have the the "second theorem of consistency for strong summability" due to Srivastava [6].

When $\phi(t)=e^{t}, \psi(t)=t^{-k}, \gamma(t)=1, m=1$, we have the following strong Riesz summability analogue of a theorem due to Tatchell [7].

THEOREM. If $k$ is a positive integer and the series $\sum a_{n}$ is summable $[R, \lambda, k]$, then $\sum a_{n} \lambda_{n}^{-k}$ is summable $\left[R, e^{\lambda}, k\right]$.

\section{REFERENCES}

1. D. Borwein and B. L. R. Shawyer, On strong Riesz summability factors, J. London Math. Soc. 40 (1965), 111-126. 
2. G. H. Hardy, The second theorem of consistency for summable series, Proc. London Math. Soc. (2) 15 (1916), 72-78.

3. G. H. Hardy and M. Riesz, The general theory of Dirichlet's series, Cambridge Tracts in Math., No. 18, Cambridge Univ. Press, England.

4. K. A. Hirst, On the second theorem of consistency in the theory of summability by typical means, Proc. London Math. Soc. (2) 33 (1932), 353-366.

5. P. Srivastava, On strong Rieszian summability of infinite series, Proc. Nat. Inst. Sci. India Part A 23 (1957), 58-71.

6. - On the second theorem of consistency for strong Riesz summability, Indian J. Mech. Math. 1 (1958-1959), 1-16.

7. J. B. Tatchell, A theorem on absolute Riesz summability, J. London Math. Soc. 29 (1954), 49-59.

8. C. J. de la Vallée-Poussin, Cours d'analyse infinitésimale, Louvain, Paris, 1923.

Wayne State University and

OAKLAND UNIVERSITY 